\title{
Fatigue mapping based on electromyography and heart rate behavior after single treadmill test
}

\begin{abstract}
Electromyography is one of the tools to measure fatigue indirectly. Widely research on electromyography and fatigue have been done include onset of fatigue and transition of fatigue during physical activity. The investigation not focusing on the muscle only, but other physiological indicator also involved to see the correlation. However, the information of electromyography behavior after activity is very limited. This paper provides investigation on lower extremities muscle activities before and after a single treadmill test. Fatigue mapping is done for electromyography and heart rate as physiological indicators. Two analyses based on time and time-frequency are chosen to process the signals. The results show that the behavior of electromyography amplitude and heart rate are back to normal condition (recover) in two to three hours, and median frequency shows faster recovery.
\end{abstract}

Keyword: Biomechanics; Electromyography; Fatigue; Medical signal processing; Timefrequency analysis 